\title{
Paper
}

\section{Expression of Subjective Evaluation for the Participation Attitude to the Internet of Male College Students Based on Visual Analog Scale}

\author{
SATOSHI WATANABE* ${ }^{\dagger}$ \\ NARUKI SHIRAHAMA \\ YuJi MATSUMOTO ${ }^{\text {II }}$ \\ YUKIO MORI**
}

\author{
Member, \\ Member, \\ Non-member, \\ Member
}

\author{
TOSHIRo TSURUtA ${ }^{\dagger}$ \\ NAOFUMI NAKAYA ${ }^{\S}$ \\ Hiroyuki TSUKAmotoll
}

\author{
Non-member \\ Member \\ Non-member
}

(Received November 8, 2019, revised January 8, 2020)

\begin{abstract}
This paper describes the expression of subjective evaluation for the participation attitude to the Internet of male college students using univariate scatter plot and box-and-whisker plot based on visual analog scale (VAS) by employing a small number of subjective evaluation measurement. 19 healthy male college students were invited as the experiment participants. In order to analyze their subjective evaluation for the participation attitude to the Internet, they were requested to answer the questionnaire on VAS (17 questions). The numerical results to show their subjective evaluation ("applicable" or "not applicable") for the participation attitude to the Internet have been obtained, and they are shown by using the combination of univariate scatter plots and box-and-whisker plots. Moreover, it has been discovered that the question based on the similar subjective evaluation belongs to the same cluster. This fact is supported by the results of published article [12] analyzed in factor analysis. In addition, it has been also discovered that participants who have similar subjective evaluation values belong to the same cluster. The relationships and meanings for these questions or participants have been found by a small number of subjective evaluation measurement based on VAS.
\end{abstract}

Keywords: Subjective evaluation, Visual analog scale, Combination of univariate scatter plot and box-andwhisker plot.

\section{Introduction}

This paper describes the expression of subjective evaluation for the participation attitude to the Internet of male college students based on visual analog scale (VAS) by small samples; experiment participants are asked to answer the questionnaire about the participation attitude to the Internet by VAS. And if this method (analysis of VAS questionnaire by small samples) is useful, it can be expected to contribute to an investigation about subjective evaluation. First of all, it is shown that the reasons why VAS was adopted for the questionnaire about the participation attitude to the Internet

\footnotetext{
* Corresponding: satoshi-w@iuhw.ac.jp

School of Health Sciences at Odawara, International University of Health and Welfare

1-2-25, Shiroyama, Odawara-shi, Kanagawa, 250-8588, Japan

$\doteqdot$ Department of Creative Engineering, National Institute of Technology (KOSEN), Kitakyushu College

5-20-1, Shii, Kokuraminami-ku, Kitakyushu-shi, Fukuoka, 802-0985, Japan

$\S$ Faculty of Health Sciences, Tsukuba International University 6-20-1, Manabe, Tsuchiura-shi, Ibaraki, 300-0051, Japan

II Junior College Division, Kindai University

3-4-1, Kowakae, Higashiosaka-shi, Osaka, 577-8502, Japan

" School of Information Studies, Shizuoka Sangyo University

4-1-1, Surugadai, Fujieda-shi, Shizuoka, 426-8668, Japan

** Department of Mechanical and Electronic Engineering, Salesian Polytechnic

4-6-8, Oyamagaoka, Machida-shi, Tokyo, 194-0215, Japan
}

and why the investigation was conducted by small samples.

Studies on the subjective evaluation based on Likert Scale (LS, see Fig. 1(a)) [1] have been conducted broadly. LS is a simple discrete scale method, but it has some weak points as follows [1] [5]: (i) LS's evaluation often becomes complicated; (ii) Sometimes several biases are introduced by the following factors, such as halo effect, leniency effect, and central tendency; and (iii) LS's results are discrete data.

From these points, it is suggested that there exists unintended bias on LS's results. In order to solve these weak points, VAS (see Fig. 1(b)) is investigated on the subjective evaluation [6] [7] VAS is a ratio scale method, it is expected that it can solve the weak points of LS as mentioned above [6] [7]. And it is considered that VAS is more flexible than LS.

Therefore, we investigated on the trend of the subjective evaluation based on VAS and LS, existence of unintended bias between LS data and VAS data was discovered [6] [7].

Moreover, we also investigated on the subjective evaluation based on VAS, such as impression of music [8] [9], impression received from the response of a communication robot [10], and so on. From these investigations, it could be able to get the results that are the same with our previous studies [8] [10].

On the other hand, in general, it is necessary to lots of measurements of subjective evaluation based on LS to ob- 
Question: I am always thinking about the Internet Applicable $1 \quad 1 \quad 2 \quad 3 \quad 4 \quad 5$ Not applicable (a) LS

Question: I am always thinking about the Internet Applicable Not applicable

(b) VAS

Figure 1: Typical types of LS and VAS.

tain reliable conclusions.

However, as a health consultation activity, it needs to give an accurate result by a small number of subjective evaluation measurement. To understand these data's distribution and other characteristics, Weissgerber et al. proposes that combination of univariate scatter plot and box-and-whisker plot of them should be drawn [11]. Therefore, this proposed method [11] is applied for our investigations [8] [10].

Similar to our previous studies [8] [10], to visualize the experimental results, combination of univariate scatter plot and box-and-whisker plot [11] is used in this paper.

Under such conditions, visualization analysis method with a small number of subjective evaluation measurement based on VAS have been proposed [8] [10]. This method consists of following components: (1) Subjective evaluation measurements based on VAS (values are 0.00-1.00); (2) Calculation of basic statistics; (3) Calculation of a correlation matrix; (4) Drawing of combination of univariate scatter plot and box-and-whisker plot [11]; (5) Hierarchical clustering analysis by Ward method; and (6) Nonhierarchical clustering analysis by k-means.

In this method, relationships and meanings of subjective evaluation values can be analyzed. This fact is consistent with our senses and it is evidence that can be analyzed by a small number of subjective evaluation measurement based on VAS [8] [10].

With the above preparation, this paper describes the expression of subjective evaluation for the participation attitude to the Internet of male college students based on VAS by a small number.

The remainder of this paper is organized as follows. Section 2 describes the theory of VAS and its visualization analysis. Materials and methods are shown in Section 3. Results and discussion are given in Section 4. Section 5 shows the conclusions and future works.

\section{Theory of Subjective Evaluation Based on VAS and its Expression}

2.1 VAS Recently, VAS has been applied to study on the subjective evaluation such as education [6] [7] [10], impression of music [8] [9] and so on.

This paper first introduces VAS. Fig. 1(b) shows typical types of VAS's questionnaire. This is to ask the participation attitude to the Internet. To answer Fig. 1(b), the response is marked by drawing a vertical line on the horizontal line. An example is shown in Fig. 2.

Calculation of VAS data is shown in Fig. 2. VAS's results are determined by the ratio of (a) to (b) (in other words, it is

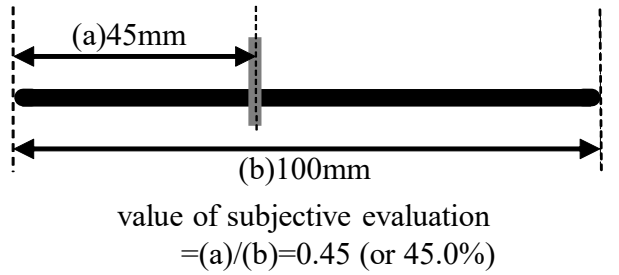

Figure 2: Example of calculation of VAS data.

determined by the position of the mark on the line)

As mentioned above, a value of subjective evaluation (between 0.00 to 1.00 ) can be obtained.

\subsection{Expression of Subjective Evaluation Based on VAS}

[8] [11] By the visualization of values obtained in 2.1, it is possible to know their characteristics. To clarify their characteristics, the analysis is carried out by $\mathrm{R}$ environment.

First, the basic statistics and a correlation matrix are calculated, the outlines of distribution and relationships of subjective evaluation values are obtained. Next, combination of univariate scatter plots and box-and-whisker plots [11] are drawing, this is our unique method [8] [10]. Moreover, hierarchical clustering analysis by Ward method and nonhierarchical clustering analysis by k-means are carried out.

By applying these procedures, relationships and meanings of subjective evaluation values and clustering of participants can be understood as the same with our previous studies [8] [10].

\section{Materials and Methods}

\subsection{Expression of Subjective Evaluation Based on VAS}

To analyze participants'subjective evaluation for the participation attitude to the Internet, they are asked to answer the questionnaire on VAS.

All participants were invited to answer questionnaire relating to the sending and reception of messages on the Internet first (see Table 1, questions from Q1.1 to Q1.7), and then to answer questionnaire relating to the participation attitude to the Internet (see Table 2, questions from Q2.1 to Q2.10). These questionnaires were extracted by Tsuruta et al.'s factor analysis [12], their validity and reliability have been confirmed [12].

Moreover, to clarify their characteristics, the following components are obtained by $\mathrm{R}$ environment as mentioned in 2.2 [8] [10]: (1) Calculation of basic statistics; (2) Calculation of a correlation matrix; (3) Drawing of combination of univariate scatter plot and box-and-whisker plots [11]; (4) Hierarchical clustering analysis by Ward method; and (5) Non-hierarchical clustering analysis by k-means.

3.2 Participants 19 healthy male college students (age: $18.74 \pm 1.21$, mean \pm standard deviation) were invited as the experiment participants, and their basic properties are summarized in Table 3. 11 participants'utilization time of their devices is 4 hours or less, on the other hand, 4 participants'utilization time of their devices is 7 hours or more (For references, 18 participants use their cell-phone of modern models, 6 of them also use another device). 
Table 1: Questionnaires relating to the sending and reception of messages on the Internet for VAS (questions from Q1.1 to Q1.7).

\begin{tabular}{|c|c|}
\hline No & Question \\
\hline Q1.1 & I get worried if I receive no response to my message. \\
\hline Q1.2 & $\begin{array}{l}\text { I want to check my cell-phone many times when I } \\
\text { send my message. }\end{array}$ \\
\hline Q1.3 & $\begin{array}{l}\text { I get annoyed when I am kept waiting for response of } \\
\text { my message. }\end{array}$ \\
\hline Q1.4 & $\begin{array}{l}\text { I get worried if I don't receive messages for long } \\
\text { time. }\end{array}$ \\
\hline Q1.5 & $\begin{array}{l}\text { I feel lonely if I receive no message when I check my } \\
\text { cell-phone. }\end{array}$ \\
\hline Q1.6 & $\begin{array}{l}\text { I think a problem is happened in human relations if } \\
\text { there is a human whose message response is slow. }\end{array}$ \\
\hline Q1.7 & $\begin{array}{l}\text { I think a problem may happen in human relations if } \\
\text { there are no exchange of message. }\end{array}$ \\
\hline
\end{tabular}

Table 2: Questionnaires relating to the participation attitude to the Internet for VAS (questions from Q2.1 to Q2.10)

\begin{tabular}{|c|c|}
\hline No & Question \\
\hline Q2.1 & I am always thinking about the Internet. \\
\hline Q2.2 & I get worried if I don't continue to use the Internet. \\
\hline $\mathrm{Q} 2.3$ & I get worried if I receive no response to my message. \\
\hline Q2.4 & $\begin{array}{l}\text { I want to check my cell-phone many times when I } \\
\text { send my message. }\end{array}$ \\
\hline Q2.5 & $\begin{array}{l}\text { Although after midnight, I would continue to use the } \\
\text { Internet for } 1 \text { hour or more. }\end{array}$ \\
\hline Q2.6 & $\begin{array}{l}\text { My sleep time becomes shorter if my utilization time } \\
\text { for the Internet is longer. }\end{array}$ \\
\hline Q2.7 & I use my cell-phone while walking or cycling. \\
\hline Q2.8 & $\begin{array}{l}\text { I want to send a message or to use the Internet, al- } \\
\text { though I converse with a person. }\end{array}$ \\
\hline Q2.9 & $\begin{array}{l}\text { I'm good at communication via the Internet than } \\
\text { face-to-face communication. }\end{array}$ \\
\hline Q2.10 & $\begin{array}{l}\text { I want to avoid the face-to-face communication be- } \\
\text { cause my main communication is via the Internet. }\end{array}$ \\
\hline
\end{tabular}

Ethical considerations are as follows: explanation of this
Table 3: Basic properties of participants

\begin{tabular}{|c|c|c|}
\hline No & Age & $\begin{array}{l}\text { Utilization time of } \\
\text { participants' devices } \\
\text { *Time/hour }\end{array}$ \\
\hline 1 & 18 & 6 \\
\hline 2 & 18 & 4 \\
\hline 3 & 20 & 4 \\
\hline 4 & 18 & 2 \\
\hline 5 & 18 & 8 \\
\hline 6 & 19 & 2 \\
\hline 7 & 19 & 3 \\
\hline 8 & 18 & 3 \\
\hline 9 & 19 & 6 \\
\hline 10 & 18 & 11 \\
\hline 11 & 18 & 12 \\
\hline 12 & 18 & 6 \\
\hline 13 & 19 & 3 \\
\hline 14 & 18 & 7 \\
\hline 15 & 18 & 3 \\
\hline 16 & 23 & 3 \\
\hline 17 & 20 & 3 \\
\hline 18 & 18 & 5 \\
\hline \multirow[t]{2}{*}{19} & 19 & 3 \\
\hline & $18.74 \pm 1.21^{* *}$ & $4.95 \pm 2.80 * *$ \\
\hline
\end{tabular}

All participants are male college student.

*Cell-phone, Personal computer, Tablet terminals, etc.

$* *$ Mean \pm Standard deviation.

study was given orally, and the documents were distributed to the participants. The participation in this study was voluntary. Furthermore, the data has been collected anonymously, and has been only used for the presentation of this study.

\section{Results and Discussion}

4.1 Basic Statistics and Correlation Matrix Table 4(a) shows the basic statistics of questionnaires relating to the sending and reception of messages based on VAS, from Q1.1 to Q1.7. All maximum values are 0.81 or more, otherwise, all minimum value is 0.00 . Therefore, distributions

Table 4: Basic statistics

(a) Q1.1 to Q1.7

\begin{tabular}{cccccccc}
\hline & Q1.1 & Q1.2 & Q1.3 & Q1.4 & Q1.5 & Q1.6 & Q1.7 \\
\hline Max & 0.88 & 0.85 & 0.78 & 0.98 & 0.98 & 0.87 & 0.81 \\
Min & 0.00 & 0.00 & 0.00 & 0.00 & 0.00 & 0.00 & 0.00 \\
Mean & 0.29 & 0.37 & 0.24 & 0.26 & 0.26 & 0.23 & 0.14 \\
Median & 0.19 & 0.31 & 0.19 & 0.14 & 0.12 & 0.11 & 0.03 \\
SD* & 0.29 & 0.27 & 0.25 & 0.30 & 0.29 & 0.29 & 0.26 \\
\hline
\end{tabular}

(b) Q2.1 to Q2.10

\begin{tabular}{ccccccccccc}
\hline & Q2.1 & Q2.2 & Q2.3 & Q2.4 & Q2.5 & Q2.6 & Q2.7 & Q2.8 & v2.9 & v2.10 \\
\hline Max & 0.67 & 0.94 & 0.88 & 0.81 & 1.00 & 1.00 & 0.73 & 0.96 & 1.00 & 0.97 \\
Min & 0.00 & 0.00 & 0.00 & 0.00 & 0.00 & 0.02 & 0.00 & 0.00 & 0.00 & 0.00 \\
Mean & 0.21 & 0.17 & 0.29 & 0.28 & 0.55 & 0.66 & 0.35 & 0.46 & 0.46 & 0.22 \\
Median & 0.14 & 0.05 & 0.12 & 0.11 & 0.75 & 0.69 & 0.32 & 0.51 & 0.38 & 0.04 \\
SD* & 0.05 & 0.08 & 0.10 & 0.08 & 0.15 & 0.08 & 0.08 & 0.09 & 0.15 & 0.12 \\
\hline
\end{tabular}

*Standard deviation. 
Table 5: Correlation matrix

(a) Q1.1 to Q1.7

\begin{tabular}{ccccccc}
\hline & Q1.1 & Q1.2 & Q1.3 & Q1.4 & Q1.5 & Q1.6 \\
\hline Q1.2 & 0.36 & - & - & - & - & - \\
Q1.3 & $\mathbf{0 . 6 2}$ & 0.39 & - & - & - & - \\
Q1.4 & 0.35 & 0.15 & 0.27 & - & - & - \\
Q1.5 & 0.05 & 0.36 & 0.08 & $\mathbf{0 . 7 9}$ & - & - \\
Q1.6 & 0.39 & 0.16 & 0.13 & 0.08 & 0.02 & - \\
Q1.7 & 0.27 & -0.31 & 0.34 & 0.26 & -0.12 & 0.41 \\
\hline
\end{tabular}

(b) Q2.1 to Q2.10

\begin{tabular}{cccccccccc}
\hline & Q2.1 & Q2.2 & Q2.3 & Q2.4 & Q2.5 & Q2.6 & Q2.7 & Q2.8 & Q2.9 \\
\hline Q2.2 & $\mathbf{0 . 7 6}$ & - & - & - & - & - & - & - & - \\
Q2.3 & 0.09 & 0.13 & - & - & - & - & - & - & - \\
Q2.4 & 0.25 & 0.01 & 0.43 & - & - & - & - & - & - \\
Q2.5 & 0.06 & -0.34 & 0.02 & -0.19 & - & - & - & - & - \\
Q2.6 & 0.49 & 0.27 & 0.01 & 0.17 & 0.39 & - & - & - & - \\
Q2.7 & -0.18 & -0.15 & 0.08 & 0.20 & 0.04 & 0.22 & - & - & - \\
Q2.8 & 0.27 & 0.26 & 0.22 & -0.18 & 0.41 & 0.43 & -0.07 & - & - \\
Q2.9 & 0.11 & 0.20 & -0.01 & 0.40 & -0.60 & -0.07 & -0.29 & -0.12 & - \\
Q2.10 & 0.30 & 0.41 & -0.31 & 0.00 & -0.38 & -0.16 & -0.52 & 0.07 & $\mathbf{0 . 7 0}$ \\
\hline
\end{tabular}

of these values are full range of these scales, and these are found to be large variation. However, other features are not clear.

On the other hand, Table 4(b) shows the basic statistics from Q2.1 to Q2.10. From Table 4(b), distributions of Q2.1 to $\mathrm{Q} 2.10$ are regarded as full range of these scales, and these are found to be large variation too. It is observed that medians of Q2.2, Q2.5, Q2.6 and Q2.10 are symmetric and characteristic. However, other features are not clear as same as Table 4(a).

In addition, the correlation matrix of these question is shown in Table 5(a). It is found that Q1.1 is moderately related to Q1.3 ( $\mathrm{r}=0.62)$ and Q1.4 is also moderately related to Q1.5 ( $\mathrm{r}=0.79)$ from Table 5(a). It is found that other correlations are weak.

Moreover, the correlation matrix from Q2.1 to Q2.10 is also shown in Table 5(b). From Table 5(b), Q2.1 is moderately related to Q2.2 ( $\mathrm{r}=0.76)$, and Q2.9 is also moderately related to $\mathrm{Q} 2.10(\mathrm{r}=0.70)$. It is found that other correlations are weak as same as Table 5(a).

That is, it is considered that relationships and meanings of subjective evaluation values or personalities of participants are not expressed from these components.

4.2 Combination of Univariate Scatter Plots and Boxand-Whisker Plots To clarify relationships and meanings of Q1.1 to Q1.7, combination of univariate scatter plots and box-and-whisker plots [11] are shown as Fig. 3(a). These distributions are found to be large variation, it can be observed that distribution of Q1.7's values is characteristic. Most of them are close to 0.00 , and all outliers of them are greater than 0.58 . This fact is the same with the results and discussion in Section 4.1.

As same as Fig. 3(a), to clarify relationships and meanings from $\mathrm{Q} 2.1$ to $\mathrm{Q} 2.10$, combination of univariate scatter plots and box-and-whisker plots [11] are also shown in
Fig. 3(b). Note that their features of distribution are different from Fig. 3(a)'s distribution.

However, these distributions are also found to be large variation, and it is observed that distribution of Q2.2, Q2.5, Q2.6 and Q2.10 are characteristic. That is, all outliers of Q2.2 and Q2.10 are greater than 0.46, and their medians are lower than 0.05 . On the other hand, medians of Q2.5 and Q2.6 are over 0.69 . These facts are evidences of the results and discussion of Section 4.1.

In contrast, relationships and meanings of Q1.1 to Q1.7 and Q2.1 to Q2.10 are not clear. This observation is same as part of our previous studies [8] [10].

\subsection{Hierarchical Clustering Analysis by Ward Method} and Non-Hierarchical Clustering analysis by k-means for VAS questionnaire Under above results and discussion, to understand relationships and meanings of subjective evaluation values and clustering of participants as consistent with our previous studies [8] [10], hierarchical clustering analysis by Ward method and non-hierarchical clustering analysis by k-means are carried out.

Figure 4 shows the cluster dendrogram from Q1.1 to Q1.7. It is considered that these are divided into three clusters from Fig. 3(a) and Tsuruta et al.'s factor analysis [12]. Furthermore, the number of clusters is defined as 3, nonhierarchical clustering analysis by k-means are also carried out. This result is same as in Fig. 4, clustering from Q1.1 to Q1.7 and their meanings [12] are summarized in Table 6. In this way, relationships and meanings of subjective evaluation values of Q1.1 to Q1.7 are visualized and understood [8] [10].

Figure 5 shows the cluster dendrogram from Q2.1 to Q2.10. It is considered that these are divided into two clusters from Fig. 3(b) and Tsuruta et al.'s factor analysis [12]. Moreover, the number of clusters is defined as 2, nonhierarchical clustering analysis by k-means are also carried 


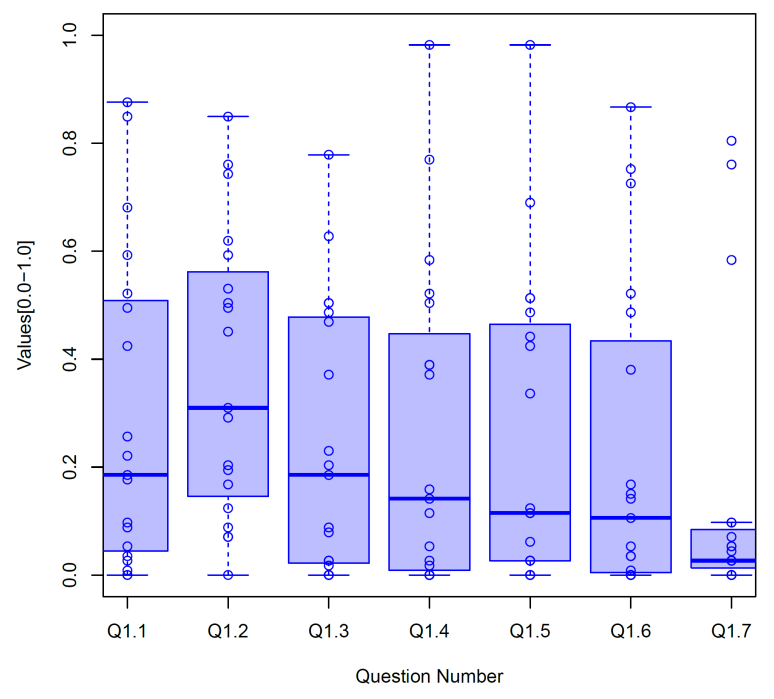

(a) Q1.1 to Q1.7

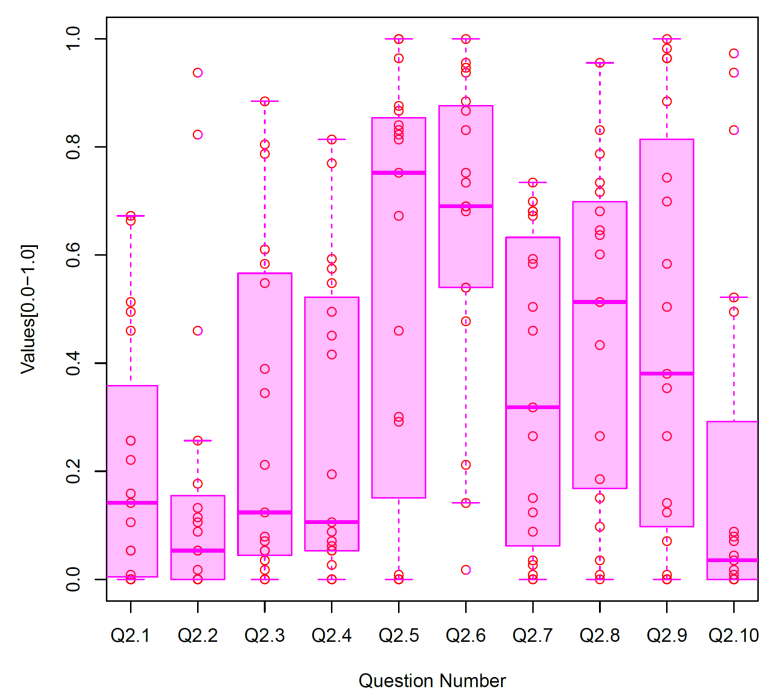

(b) Q2.1 to Q2.10

Figure 3: Combination of univariate scatter plots and box-and-whisker plots.

out. This result is same as in Fig. 5, clustering from Q2.1 to Q2.10 and their meanings [12] are summarized in Table 7. As same as Fig. 4 and Table 6, relationships and meanings of subjective evaluation values of Q2.1 to Q2.10 are also
Cluster Dendrogram

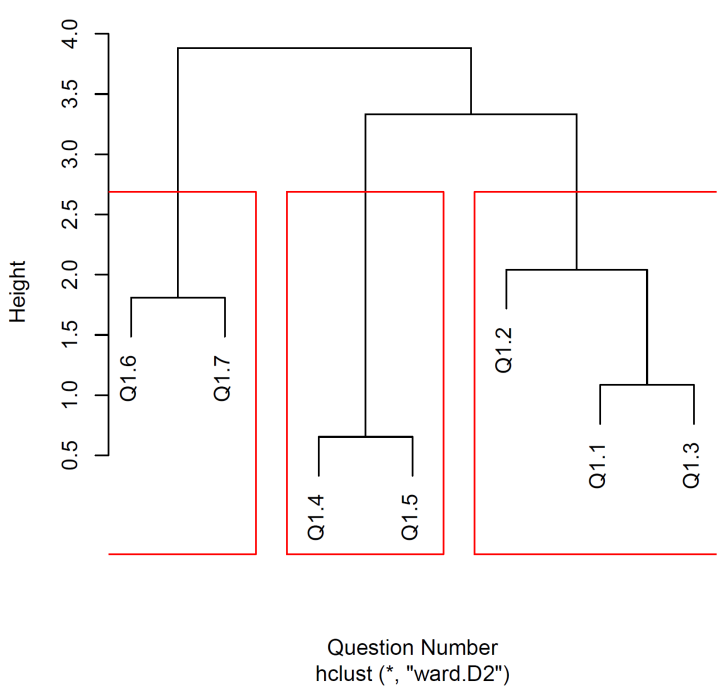

Figure 4: Cluster dendrogram from Q1.1 to Q1.7.

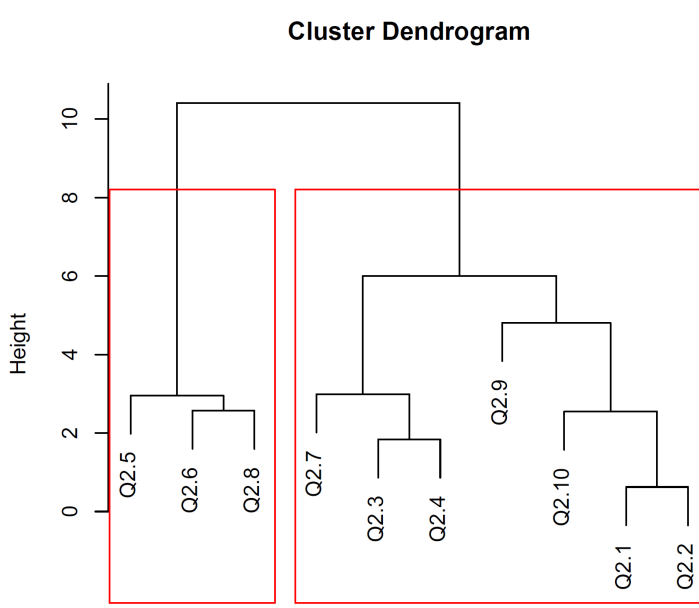

Question Number hclust (*. "ward.D2")

Figure 5: Cluster dendrogram from Q2.1 to Q2.10.

visualized and understood [8] [10].

As mentioned above, relationships and meanings of subjective evaluation values can be visualized and analyzed.

Table 6: Clustering by k-means from Q1.1 to Q1.7 and their meanings.

\begin{tabular}{cl}
\hline Cluster & Meaning \\
\hline Q1.4, Q1.5 & Questions to seek sympathy for the Internet. \\
Q1.1, Q1.3, Q1.2 & Questions related to emotional stability for response of message. \\
Q1.6, Q1.7 & Questions about human relations. \\
\hline
\end{tabular}

Table 7: Clustering by k-means from Q2.1 to Q2.10 and their meanings.

\begin{tabular}{cl}
\hline Cluster & Meaning \\
\hline Q2.1, Q2.2, Q2.10, Q2.9, Q2.3, Q2.4, Q2.7 & Utilization, attitude and communication for the Internet \\
Q2.6, Q2.8, Q2.5 & Priority of utilization for the Internet. \\
\hline
\end{tabular}


Table 8: Clustering by k-means of participants based on their values from Q1.1 to Q1.7 and their utilization time of their devices (median).

\begin{tabular}{lc}
\hline Clustering and participants'No & $\begin{array}{l}\text { Utilization time of } \\
\text { participants'devices } \\
\text { (median,hour) }\end{array}$ \\
\hline Cluster 1: 1, 2, 3, 4, 14,15,16,17 & 3.5 \\
Cluster 2: 5, 6, 8, 9,10,11,19 & 6 \\
Cluster 3: 7, 12,13,18 & 4 \\
\hline
\end{tabular}

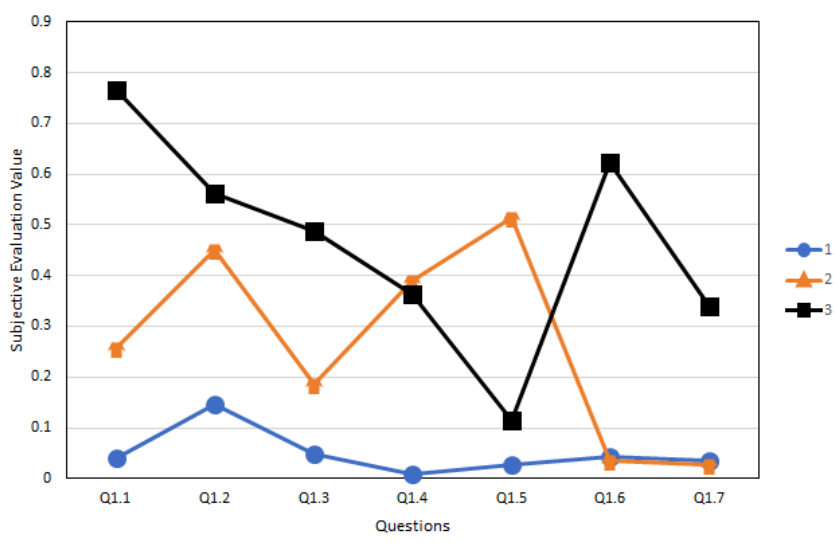

Figure 6: Medians from Q1.1 to Q1.7 for the participants who are belong to the same cluster (1).

This fact is consistent with our senses and it is evidence that can be analyzed by a small number of subjective evaluation measurement based on VAS [8] [10].

\subsection{Non-Hierarchical Clustering analysis by k-means} for Participants Under the same conditions as section 4.3 (the number of clusters is defined as 3), clustering of participants is also obtained by non-hierarchical clustering analysis based on k-means. These clustering are shown in Table 8, and their utilization times of their devices (median) are also shown in Table 8.

To understand these meanings, it is calculated that the median of subjective evaluation value (i.e., from Q1.1 to Q1.7) for the participants who are belong to the same cluster. These are plotted in Fig. 6, medians of cluster 1 are generally low, on the other hand, medians of cluster 3 are higher than medians of cluster 1 and 2 (expect for Q1.4 and Q1.5).

Here, Fig. 6 is divided by the Table 6's clusters (from Q1.1 to Q1.7's cluster), they are represented as in Fig. 7. In Fig. 7(b) and Fig. 7(c), medians of cluster 3 are higher than medians of cluster 1 and 2. Especially, medians of cluster 1 and 2 are very low in Fig. 7(c) (close to 0.00). However, the relationships between these facts and participants'utilization time of their devices are not clear (see Table 7).

By the way, it can be seen that median of cluster 2 are higher than the median of cluster 1 and that of cluster 3 in Fig. 7(a). It is suggested that the observation in Fig. 7(a) and their utilization time of their devices are related (see Table $8)$.

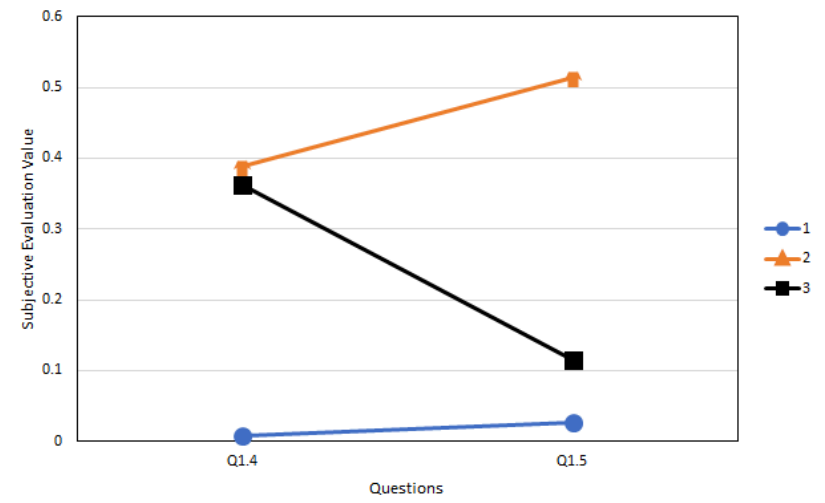

(a) Cluster 1

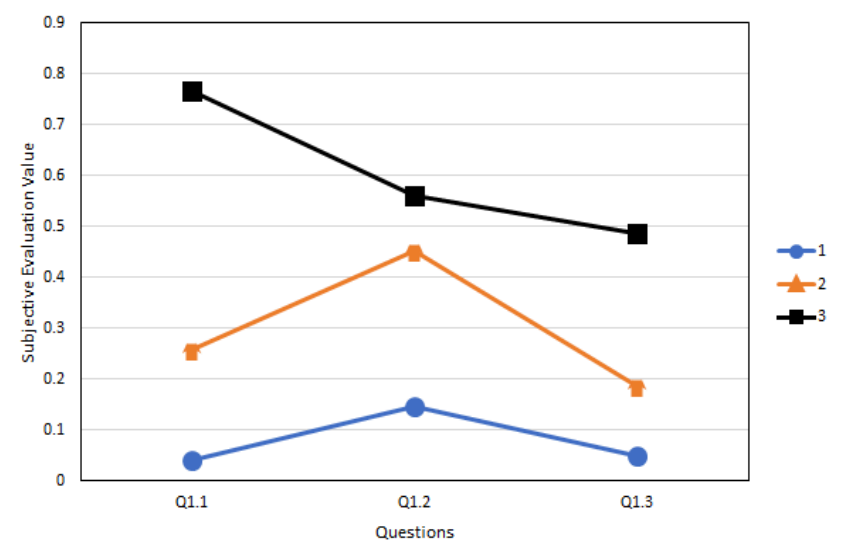

(b) Cluster 2

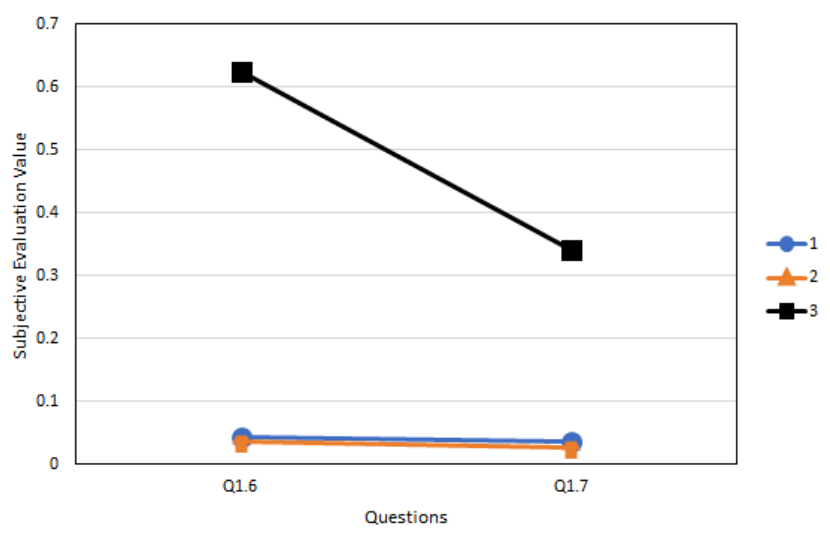

(c) Cluster 3

Figure 7: Medians from Q1.1 to Q1.7 for the participants who are belong to the same cluster (2) (Fig. 6. is divided by the Table 6's (questions') clusters).

As same as Table 8 and Fig. 6, clustering of participants based on Q2.1 to Q2.10 is also obtained. These clustering and their utilization time of their devices (median) are shown in Table 9. Note that the number of clusters and member of same cluster are different from Table 8 .

On the other hand, as same as Fig. 6, it is calculated that the median of subjective evaluation value (i.e., from Q2.1 to Q2.10) of the participants who are belong to the same cluster. Although these are plotted in Fig. 8, feature of that is not clear. 
Table 9: Clustering by k-means of participants based on their values from Q2.1 to Q2.10 and their utilization time of their devices (median).

\begin{tabular}{lc}
\hline Clustering and participants'No & $\begin{array}{l}\text { Utilization time of } \\
\text { participants'devices } \\
\text { (median,hour) }\end{array}$ \\
\hline Cluster 1: 1, 5, 11, 12,13,16,19 & 6 \\
Cluster 2: 2, 3, 4, 6, 7,8,9,10, & 3.5 \\
$14,15,17,18$ & \\
\hline
\end{tabular}

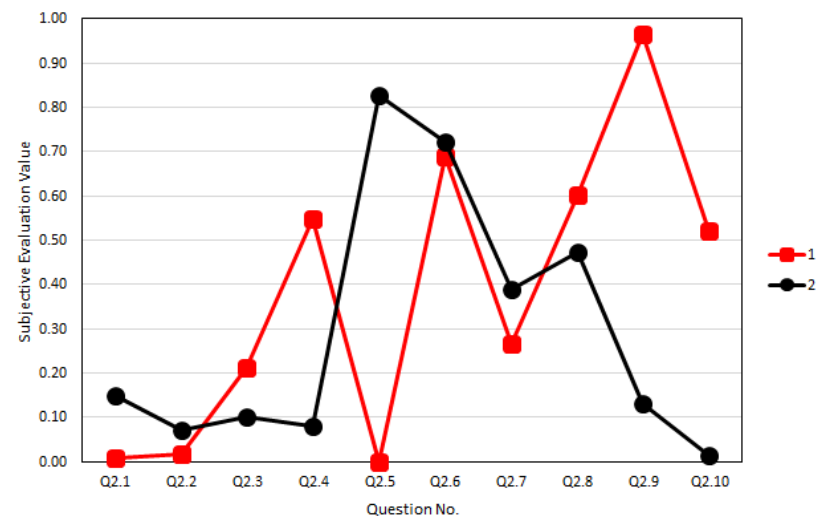

Figure 8: Median of values from Q2.1 to Q2.10 for the participants who are belong to the same cluster.

In addition, relationships between participants'utilization time of their devices and these facts are also not clear. Therefore, Q2.4, Q2.5, Q2.9 and Q2.10 are focused, because their differences are large (0.47 or higher). In Q2.4, Q2.9 and Q2.10, medians of cluster 1 are larger than medians of cluster 2 . On the other hand, median of cluster 1 is smaller than median of cluster 2 in Q2.5.

It is be considered that these differences and between participants'utilization time of their devices are related (see Table 9). Especially in Q2.5, it can be also considered that median of cluster 2 is higher because the utilization time of cluster 2's participants'devices is shorter (see Table 9, that is, because of the utilization time of cluster 2's participants'devices is shorter, then they would be thought and tried that "Although after midnight, I would continue to use the Internet for 1 hour or more".).

As mentioned above, relationships and meanings of participants can also be visualized and understood by using results of clustering analysis for questionnaires and participants.

\section{Conclusions}

To analyze the relationships and meanings of subjective evaluation, this work carried out the visualizations of subjective evaluation for the participation attitude to the Internet of male college students by a small number of subjective evaluation measurement based on VAS. This method consists of following three procedures: calculation of basic statistics and a correlation matrix; drawing of combination of univariate scatter plots and box-and-whisker plots [11]; and hierarchical clustering analysis by Ward method and non-hierarchical clustering analysis by k-means.

Under these procedures, the relationships and meanings of subjective evaluation and participants can be visualized and understood, they are consistent with our previous studies and published article[8] [10] [12].

By the way, in general, it is necessary to use large measurements of subjective evaluation to obtain reliable conclusions. On the other hand, this proposed method is very useful to analyze by a small number of subjective evaluation measurement based on VAS.

To obtain reliable conclusions, applying this method in the future study and conducting more investigation are very important.

Future works are as follows: (1) clustering of participants in details, (2) measurement with larger number of participants, (3) investigation of differences in LS measurement and VAS measurement, and (4) applying this method in another fields.

\section{Acknowledgment}

This work is partially supported by JSPS Grants-in-Aid for Scientific Research, No.16K01890.

\section{References}

[1] Likert, R., "A technique for the measurement of attitudes", Archives of Psychology (New York), 1932.

[2] Peabody, D., "Two components in bipolar scales: Direction and extremeness", Psychological Review, Vol.69, No.2, pp.65-73, 1962. DOI: 10.1037/h0039737

[3] Upshaw, H. S., "Own attitude as an anchor in equalappearing intervals", The Journal of Abnormal and Social Psychology, Vol.64, No.2, pp.85-96, 1962. DOI: 10.1037/h0048600

[4] Cummins, R. A., "The Second Approximation to an International Standard for Life Satisfaction”, Social Indicators Research, Vol.43, No.3, pp.307-334, 1998. DOI: 10.1023/A:1006831107052

[5] Heine, S. J., Lehman, D. R, Peng, K. and Greenholtz, J., "What's wrong with cross-cultural comparisons of subjective Likert scales?: The reference-group effect", Journal of Personality and Social Psychology, Vol.82, No.6, pp.903-918, 2002. DOI: $10.1037 / 0022-3514.82 .6 .903$

[6] Watanabe, S., Shirahama, N., Tsukamoto, H., Matsumoto, Y., Nakagawa, M., Miyamoto, K., Nakaya, N., Tomita, M. and Mori Y., "Trend of the Subjective Evaluation based on Visual Analog Scale and Likert Scale", ICIC Express Letters, Part B: Applications (ICIC-ELB), Vol. 7, No.1, p.37-42, 2016.

[7] Watanabe, S., Shirahama, N., Matsumoto, Y., Tsukamoto, H., Nakagawa, M., Miyamoto, K., Nakaya, N., Tomita, M. and Mori, Y., "Distribution Trend of the Information Skills for Students Based on Visual Analog Scale and Likert Scale", International Journal of Biomedical Soft Computing and Human Sciences, Vol.20, No.2, pp.1-6, 2016. DOI: 10.24466/ijbschs.20.2_1 
[8] Watanabe, S., Shirahama, N., Matsumoto, Y., Tsukamoto, H., Nakaya, N. and Mori, Y., "Subjective Evaluation for the Impression of Music Based on Visual Analog Scale and Cluster Analysis", Journal of Biomedical Fuzzy Systems Association, Vol.19, No.2, pp.53-65, 2017 (in Japanese).

[9] Watanabe, S., Shirahama, N., Matsumoto, Y., Tsukamoto, H., Nakaya, N. and Mori, Y., "Proposal of a New Concept of Subjective Evaluation Measurement Based on Visual Ana$\log$ Scale", Proceedings of the 30th Annual Conference of Biomedical Fuzzy Systems Association, pp.157-160, 2017(in Japanese).

[10] Shirahama, N., Watanabe, S., Ikegami, F., Nakaya N. and Mori, Y., "A Study on the Impression Received from the Response of a Communication Robot", Proceedings of the 5th IIAE International Conference on Intelligent Systems and Image Processing 2017 (ICISIP 2017), pp.356-363, 2017. DOI: $10.12792 /$ icisip2017.065

[11] Weissgerber, T. L., Milic, N. M., Winham, S. J. and Garovic, V. D., "Beyond Bar and Line Graphs: Time for a New Data Presentation Paradigm", PLOS Biology, Vol.13, No.4, 2015. DOI: 10.1371/journal.pbio.1002128

[12] Tsuruta, T., Yamamoto, Y. and Nojima, E., Nakaya N. and Mori, Y., "Development of the Internet Addiction Tendency Scale for High-School Students", Japan Journal of Educational Technology, Vol.37, No.4, pp.491-504, 2014 (in Japanese).

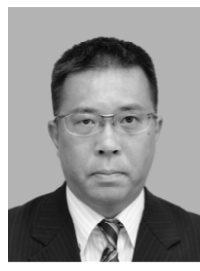

Satoshi Watanabe (Member) received his $\mathrm{Ph} . \mathrm{D}$. degree in information system from University of East Asia, Shimonoseki, in 2008. He is currently an associate professor at School of Health Sciences at Odawara, International University of Health and Welfare. His current research interests are informatics, human science and educational technology. $\mathrm{He}$ is a member of IIAE, BMFSA, ASJ, IPSJ, JSET and so on.

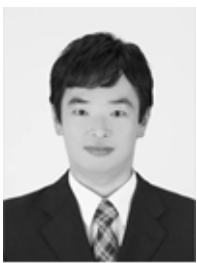

Toshiro Tsuruta (Non-member) received his Ph.D. degree form Waseda University in 2015. $\mathrm{He}$ is currently an assistant professor at School of Health Sciences at Odawara, International University of Health and Welfare. His current research interests are prevention education for the Internet addiction and educational technology. He is a member of BMFSA, JSET, JAEIS,

NASEM and so on.

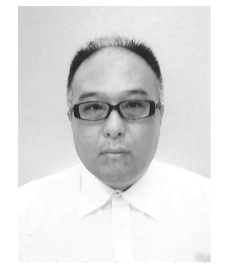

Naruki Shirahama (Member) received his Ph.D. degree in electrical engineering from Kyushu Institute of Technology in 2000. He has been working as a professor at National Institute of Technology (KOSEN), Kitakyushu College. His present research interests are include the application of subjective observation model and so on. He is a member of IIAE and BMFSA.

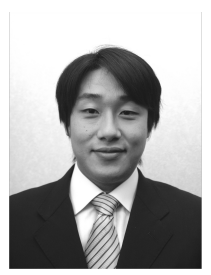

Naofumi Nakaya(Member) received his Ph.D. degree from Nihon University in 2015. $\mathrm{He}$ is currently an assistant professor at Tsukuba International University. $\mathrm{He}$ is a member of IIAE and BMFSA.

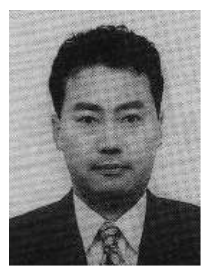

Yuji Matsumoto (Non-member) received his Ph.D. degree from Osaka University. $\mathrm{He}$ is curently a professor at Junior College Division, Kindai University. He is a member of BMFSA.

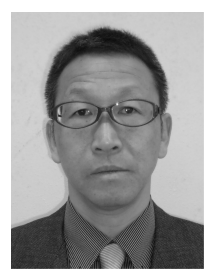

Hiroyuki Tsukamoto (Non-member) received his B.E. degree from Yokohama National University. He is curently a professor at Shizuoka Sangyo University. He is a senior coach of Japan Sports Association and Japan Volleyball Association.

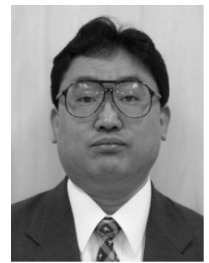

Yukio Mori (Member) received his Ph.D. degree from Tokyo University of Science. He has been working as a professor at Department of Mechanical and Electronic Engineering, Salesian Polytechnic. He is a member of IIAE and BMFSA. 